\title{
Influence of structured telephone follow-up on patient compliance with rehabilitation after total knee arthroplasty
}

This article was published in the following Dove Press journal:

Patient Preference and Adherence

3 March 2016

Number of times this article has been viewed

\section{Mochuan Chen' \\ Pihong $\mathrm{Li}^{2}$ \\ Feiou $\operatorname{Lin}^{3}$}

'Department of Orthopaedics, ${ }^{2}$ Department of General Surgery, The Second Affiliated Hospital \& Yuying Children's Hospital, ${ }^{3}$ Orthodontic Department, School and Hospital of Stomatology, Wenzhou Medical University, Wenzhou, Zhejiang,

People's Republic of China
Correspondence: Feiou Lin Orthodontic Department, School and Hospital of Stomatology, Wenzhou Medical University, No 373, West Xueyuan Road, Wenzhou, Zhejiang 325000, People's Republic of China

Tel/fax +865778806 3008

Email linfeiousci@I63.com
Introduction: To assess the effects of structured telephone follow-up on patient's home-exercise compliance after total knee arthroplasty (TKA).

Methods: A total of 202 elderly patients who received a unilateral TKA were enrolled in this study. The participants were randomized into two groups: the intervention group that received structured telephone follow-up after discharge and the control group that received routine health care. Pain, functional ability, quality of life, and depression survey scores were measured before and after TKA. The intergroup and intragroup differences were analyzed during the 12 months following discharge.

Results: There were no significant differences in the sociodemographic characteristics of both groups. The mean home-exercise time and total days in the intervention group were significantly higher than those in the control group. Variable scores differed significantly between groups. Pain, functional ability, quality of life, and depression improved significantly after TKA in both groups, and the intervention group had greater improvement in mental health and active range of motion.

Conclusion: Undergoing a TKA can significantly reduce the patient's pain from osteoarthritis while improving the overall physical function and quality of life. Furthermore, a structured telephone follow-up can improve patient adherence to home exercise after TKA.

Keywords: quality of life, pain, home exercises, patient-reported outcomes

\section{Introduction}

The main complaints of patients with osteoarthritis include pain and a restricted range of motion (ROM). Deterioration in function accompanied by increasing pain ultimately affects the patient's social and emotional well-being. Relieving pain and improving physical function and quality of life are the main benefits of total knee arthroplasty (TKA). ${ }^{1,2}$ TKAs have become common orthopedic procedures and are effective in the management of severe knee osteoarthritis. It was demonstrated that TKA effectively eliminated knee pain caused by degenerative joint disease. ${ }^{3,4}$ The number of TKAs performed in the People's Republic of China has recently grown due to the aging population and advances in surgical and anesthetic techniques. Most patients who undergo TKAs are between the ages of 50 years and 80 years, and $85 \%$ of patients report being satisfied with their outcome. ${ }^{5}$

Despite this satisfaction, the functional benefits of TKAs are not convincing. Many patients continue to demonstrate functional, strength, and mobility deficits after a TKA. ${ }^{6-8}$ During stair ascent and descent, women took nearly twice the time and were $30 \%$ weaker than women without knee pathology 1 year after surgery. 
These differences were even greater for men who received TKA. Despite the significant improvement in the patients' health-related quality of life after TKA, post-TKA healthrelated quality of life was still significantly lower than that in a healthy matched population. ${ }^{9,10}$ A systematic Cochrane review showed no studies providing direct evidence that multidisciplinary rehabilitation following TKA had improved patient outcomes compared with controls. However, five trials included in this review provided evidence that multidisciplinary rehabilitation can improve patient activity and participation in therapy. ${ }^{11}$ Short- and long-term outcomes after TKA may be associated with the type and intensity of postoperative rehabilitation the patients received. Several accepted rehabilitation protocols for patients after TKA have been reported in the literature. ${ }^{11}$

In the People's Republic of China, rehabilitation after TKA usually relies on outpatient therapy and self-motivated exercises, rather than on long in-hospital stays. The length of stay in the hospital has decreased annually, and more patients were discharged to home-based rehabilitation. Patient compliance with home-based rehabilitation therefore becomes the key to the whole rehabilitation protocol. The objective of this study was to assess the impact of structured telephone reinforcement on patient compliance with home exercises after TKA.

\section{Methods}

The research was designed as a pilot randomized controlled trial. Participants were recruited between May 2013 and May 2014 based on the following inclusion criteria: 60-75 years old, a diagnosis of primary osteoarthritis of the knee, the patient's first unilateral TKA, residence in Wenzhou city, a body mass index of $<40$, previously ambulatory with or without a walking aid, and willing to answer questionnaires. Exclusion criteria included an associated condition impeding compliance with functional rehabilitation (neurologic disorders, severe psychiatric conditions, schizophrenia, and depression), language difficulties, postoperative or intraoperative complications, and problems understanding the instructions of the research protocol. Ethical approval was obtained from the Health Research Ethics Board at The Second Affiliated Hospital. Each patient was given oral and written information and signed the consent form before being accepted into the study.

In total, 1,500 patients received a TKA at our large academic medical center. Of these, 208 eligible patients participated in this study. Eligible patients were randomized into two groups using a computer-generated table of random numbers with a block size of ten and an allocation ratio of 1:1. The patients were sequentially allocated to treatment groups in the order in which they were recruited. The intervention assignment was allocated using sealed, opaque envelopes by a statistician. All evaluators and investigators were blinded to the patient group assignment for the duration of the study. Our sample size was sufficient enough to detect an expected difference of $15 \%$ in the maximum distance the patient can walk in 6 minutes, with a type II error of 0.20 ( $80 \%$ power) and a $P$-value $<0.05$ defined as significant.

The same treatment program was applied to all patients during their hospitalizations. The surgeries were performed by three surgeons who standardized their surgical techniques for the research study. All patients were treated according to the same standardized rehabilitation protocol. This rehabilitation protocol had already been in use at our institution prior to the start of the study, so all staff members were familiar with the treatment routine. Our rehabilitation program consisted of simple exercises to improve gait, ROM, and muscle strength, as well as to decrease pain. This included ROM exercises, quadriceps activation, and exercises to improve activities of daily living such as sitting, standing, walking, and climbing stairs. Exercise intensity was gradually increased based on the individual patient's pain and tolerance.

\section{Intervention}

A series of intervention programs to improve patient adherence to the home-exercise routine was developed and was easy to apply in clinic. The patients were educated about the reasons for home exercise that included a discussion of the benefits of home exercise and an explanation of the negative effects of poor exercise adherence after TKA. Five experienced nurses were trained to teach all patients the standardized home exercises prior to hospital discharge. A standard rehabilitation protocol manual and video were also issued to discharged patients combined with verbal information. The patients were asked to perform home exercises for 1 hour/d for 12 weeks.

After discharge, the patients received a structured telephone call that reinforced care and reassurance during therapy. One week after discharge, the first telephone call was used to check the patient's initial compliance with home exercise, the patient's well-being, pain, and discomfort; the use of analgesics; reassurance regarding the patient's reaction to normal activity; and to reinforce the patient's understanding of the importance and benefits of home exercise. Furthermore, during the call, the nurses collected details about the patient's daily home exercise, adjusted the intensity 
of the exercise, and answered any questions the patient had. If there were some complicated problems that could be solved immediately over the phone, a rehabilitation physician would review the documentation from the phone call and discuss potential solutions with the nurse. Home visits were made as necessary. The same structured telephone call was also made 3 weeks and 6 weeks after TKA. The phone call lasted 5-10 minutes on average. All telephone calls were conducted by three experienced nurses using the same protocol. All questionnaires were administered by two other nurses. All nurses were blinded to their group assignment for this study. The patients returned to the clinic for reexamination 3 months and 6 months after discharge. The control group had the same standardized rehabilitation protocol training prior to hospital discharge, but received no telephone call. Contact for the control group was limited to their 3- and 6-month follow-up.

\section{Outcome and statistical analysis}

A descriptive data questionnaire was used to collect baseline data from all patients. A $100 \mathrm{~mm}$ visual analog scale questionnaire was used to assess pain. The patients were asked to mark a location on the line corresponding to their highest experienced pain intensity, with scores ranging from no pain (0) to severe pain (100). ${ }^{12} \mathrm{ROM}$ was calculated using a $360^{\circ}$ double-armed goniometer. ${ }^{13}$ Goniometry was performed by the same nurse to ensure reliability and validity. Knee function and stiffness were measured using the Western Ontario and McMaster Universities Osteoarthritis Index (WOMAC). The WOMAC is a valid and reliable self-reported questionnaire for the patients with osteoarthritis and knee arthroplasties. ${ }^{14-17}$ It consists of 24 items on three subscales: pain, stiffness, and physical function. The WOMAC pain, physical function, and stiffness subscales comprise five, 17, and two questions, respectively. In this study, we only used the physical function and stiffness subscales. Results were evaluated out of 100 points, with " 100 " defined as "healthy" and "0" defined as "poor health". Quality of life was measured by the Short Form-36. ${ }^{18} \mathrm{~A}$ valid and reliable Chinese translation had been approved previously. ${ }^{19,20}$ The Short Form-36 questionnaire evaluates eight health domains: physical functioning, physical capacity, bodily pain, general health, vitality, social functioning, emotional capacity, and mental health. These domains are summarized into the physical component summary and the mental component summary (MCS). The Beck Depression Inventory scale was used to identify signs of depression in the patients. This scale served to identify the severity of the patient's depression, rather than diagnose depression.
The telephone intervention lasted for 12 weeks, after which there was no further intervention. All questionnaires were administered preoperatively and at 3 months, 6 months, and 12 months after discharge. A chart was given to the patients to facilitate self-monitoring of homeexercise time after discharge from the hospital. The patients were encouraged to fill out the chart on a daily basis after home exercise.

SPSS software Version 15 (SPSS Inc., Chicago, IL, USA) was used to perform statistical analysis, and a one-way analysis of variance (ANOVA) was performed using the least significant difference post hoc test to assess outcomes across groups. A repeated measures ANOVA was performed to assess the interaction effects between two groups. An independent samples $t$-test was performed to observe intragroup differences. Before conducting these tests, data were checked for normality and equality of variances using the Kolmogorov-Smirnov and Levene's tests, respectively. All variances were normal and equal. The results were evaluated within a $95 \%$ confidence interval. Statistical significance was defined as $P<0.05$.

\section{Results}

Participant demographics are shown in Table 1. Figure 1 shows the Consolidated Standards or Reporting Trials diagram of the clinical trial. A total of 15 patients (intervention group, $\mathrm{n}=7$; control group, $\mathrm{n}=8$ ) were lost to follow-up due to an invalid questionnaire or failing to review on time. The final total sample size was 187 . The mean age of the intervention group was $66.18 \pm 3.59$ years and that of the control group was $67.05 \pm 4.05$ years. There were no significant sociodemographic differences between the two groups $(P>0.05)$. Table 2 shows the mean home-exercise time for each group. The mean home-exercise time and days in the intervention group were significantly higher than those in the control group $(P<0.01)$.

Data recorded for different time periods are shown in Table 3. The pain and Beck Depression Scale scores of the intervention group were significantly lower than those of the control group $(P<0.01) 3$ months after TKA. The MCS scores of the intervention group were significantly higher than those of the control group $(P<0.01)$ after TKA. The active ROM of the intervention group was significantly higher than that of the control group $(P<0.01)$ at 6 months and 12 months after TKA. The functional difficulty experienced by the intervention group was significantly lower than that by the control group $(P<0.05) 12$ months after TKA. All variable scores differed significantly between the four time points between groups $(P<0.01)$. Figure 2 describes the individual variable 
Table I Demographic characteristics of the study participants

\begin{tabular}{|c|c|c|c|}
\hline Demographic & Intervention group & Control group & $P$-value \\
\hline Age & $66.18 \pm 3.59$ & $67.05 \pm 4.05$ & 0.191 \\
\hline $\operatorname{Sex}(n)$ & & & 0.450 \\
\hline Male & 30 & 25 & \\
\hline Female & 64 & 68 & \\
\hline Level of education & & & 0.122 \\
\hline Illiterate and primary school & 40 & 43 & \\
\hline Secondary school & 45 & 39 & \\
\hline Tertiary & 9 & 11 & \\
\hline Body mass index & $31.44 \pm 7.02$ & $29.85 \pm 7.56$ & 0.093 \\
\hline Affected side, right & 37 (39.36\%) & $4 \mathrm{I}(44.09 \%)$ & 0.512 \\
\hline Hypertension & $26(27.66 \%)$ & $24(25.81 \%)$ & 0.775 \\
\hline Cardiac problems & $14(14.89 \%)$ & $16(17.20 \%)$ & 0.667 \\
\hline Pulmonary problems & $4(4.26 \%)$ & $5(5.38 \%)$ & 0.747 \\
\hline Diabetes & $19(20.21 \%)$ & $17(18.28 \%)$ & 0.737 \\
\hline Living alone & $15(15.96 \%)$ & $13(13.98 \%)$ & 0.705 \\
\hline Total & 94 & 93 & \\
\hline
\end{tabular}

Note: Statistically significant $(P<0.05)$.

scores measured 12 months after discharge. Table 4 shows that mental health and active ROM were significantly different when intersubject effects were tested.

\section{Discussion}

Previously, supervised physiotherapy, conducted two or three times weekly, was a commonly preferred method of rehabilitation. ${ }^{21}$ However, this approach costs a significant amount of money and manpower. Some specialists decided to focus on home exercise, as it has been found to be as effective as supervised physical therapy in some studies. ${ }^{22,23}$ In the People's Republic of China, home exercise was usually recommended to the patients after knee surgery to help them return to their predisease state. Unfortunately, there are no studies evaluating patient compliance in relation to home exercises after TKA. To our knowledge, this study is the first to propose a feasible intervention to improve patient adherence to home exercise. There were no significant differences between the two groups at baseline, so the results can be interpreted more authoritatively.

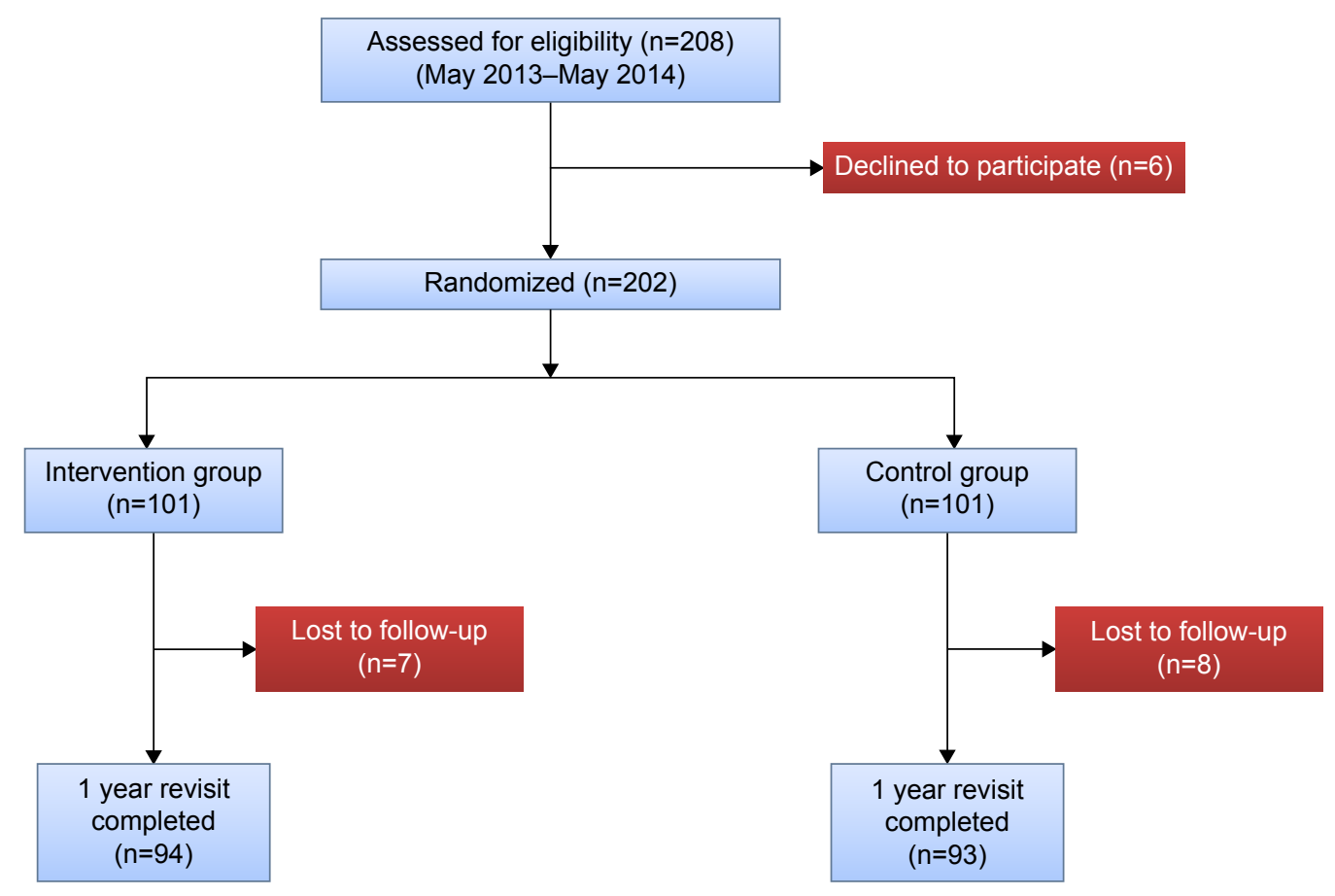

Figure I CONSORT diagram of the clinical trial.

Abbreviation: CONSORT, Consolidated Standards or Reporting Trials. 
Table 2 Mean home-exercise times in the control and telephone follow-up groups

\begin{tabular}{llll}
\hline Variable & Intervention group & Control group & $\boldsymbol{P}$-value \\
\hline Days (n) & $78.35 \pm 5.20$ & $70.21 \pm 6.05$ & $<0.01$ \\
Time (minutes) & $54.12 \pm 5.71$ & $48.95 \pm 7.21$ & $<0.01$ \\
\hline
\end{tabular}

Note: Statistically significant $(P<0.05)$.

Patik et $\mathrm{al}^{24}$ found that telephone-based outreach programs for the patients with pediatric sickle cell disease was cost effective and improved patient adherence to comprehensive care. The results of this study suggest that patient compliance to home exercises can be improved by structured telephone reinforcement. Telephone-based structured follow-up, psychosocial support, and health education have been shown to improve patient adherence and surgical outcomes for several conditions. ${ }^{25-27}$ Additionally, telephone-based nurse outreach programs were easy to adopt, implement, and maintain, with wide reach and moderate efficacy. Our study therefore chose nurse-led telephone-based interventions. In the telephone group, the ROM significantly increased and the functional difficulty significantly decreased. This might be attributed to good patient compliance. A previous study found that telephone calls from a health-care provider significantly reduced both the self-reported pain and anxiety of a sample of patients who began orthodontic treatment. ${ }^{28}$ Similarly, our study found that the self-reported pain and Beck Depression Scale scores were significantly lower at 3 months after TKA in the intervention group. This might be attributed to enhanced communication after discharge. In a study of breast-cancer patients, researchers discovered that enhanced communication between patients and health care providers improved the patients' psychological adjustment to treatment. ${ }^{29}$ Correspondingly, the MCS was significantly greater in the telephone group.

The results of our study confirm the effectiveness of TKA at improving patients' functional ability while decreasing their pain, as reported at short-term and mid-term follow-ups. This improvement was dramatic during the first 6 months in both groups. Furthermore, at 6 months and 12 months after TKA, there was a significant improvement in the pain, MCS, and function of both groups. This indicates that the first 6 months after TKA are very important for the rehabilitation.

Table 3 Patient-reported outcomes and active ROM before and after TKA

\begin{tabular}{|c|c|c|c|c|c|c|}
\hline Variable & $\begin{array}{l}\text { Before the } \\
\text { operation }\left(T_{0}\right)\end{array}$ & 3 months $\left(T_{1}\right)$ & 6 months $\left(T_{2}\right)$ & 12 months $\left(T_{3}\right)$ & $\begin{array}{l}\text { ANOVAF } \\
\text { (P-value) }\end{array}$ & $\begin{array}{l}\text { LSD post } \\
\text { hoc test }\end{array}$ \\
\hline \multicolumn{7}{|l|}{ Intervention group } \\
\hline \multicolumn{7}{|l|}{$\operatorname{ROM}\left({ }^{\circ}\right)$} \\
\hline Active & $73.5 \pm 11.9$ & $86.7 \pm 9.6$ & $101.3 \pm 10.6 *$ & $102.5 \pm 10.9 *$ & $<0.01$ & $T_{3}, T_{2}>T_{1}>T_{0}$ \\
\hline Passive & $79.9 \pm 12.5$ & $92.2 \pm 10.2$ & $107.3 \pm 9.6 * *$ & $108.1 \pm 9.4^{*}$ & $<0.01$ & $T_{3}, T_{2}>T_{1}>T_{0}$ \\
\hline \multicolumn{7}{|l|}{ WOMAC score } \\
\hline Stiffness & $74.6 \pm 14.1$ & $26.3 \pm 9.1$ & $18.6 \pm 7.1$ & $17.9 \pm 6.5$ & $<0.01$ & $T_{0}>T_{1}>T_{2}, T_{3}$ \\
\hline Function & $68.2 \pm 13.5$ & $20.7 \pm 8.2$ & $13.2 \pm 6.2$ & $13.9 \pm 7.1 *$ & $<0.01$ & $T_{0}>T_{1}>T_{3}, T_{2}$ \\
\hline Pain (VAS) & $72.2 \pm 12.4$ & $16.1 \pm 6.2 * *$ & $15.6 \pm 5.5$ & $8.7 \pm 5.1$ & $<0.01$ & $T_{0}>T_{1}, T_{2}>T_{3}$ \\
\hline Beck Depression Scale & $17.2 \pm 9.1$ & $8.1 \pm 2.7 *$ & $7.3 \pm 2.1$ & $7.9 \pm 1.9$ & $<0.01$ & $T_{0}>T_{1}, T_{3}, T_{2}$ \\
\hline \multicolumn{7}{|l|}{ SF-36 } \\
\hline PCS & $32.8 \pm 7.3$ & $39.7 \pm 7.6$ & $40.1 \pm 6.8$ & $39.4 \pm 6.7$ & $<0.01$ & $T_{2}, T_{1}, T_{3}>T_{0}$ \\
\hline MCS & $45.6 \pm 8.4$ & $55.9 \pm 6.7^{*}$ & $54.1 \pm 7.3^{*}$ & $52.5 \pm 7.5 * *$ & $<0.01$ & $T_{1}, T_{2}>T_{3}>T_{0}$ \\
\hline \multicolumn{7}{|l|}{ Control group } \\
\hline \multicolumn{7}{|l|}{$\operatorname{ROM}\left({ }^{\circ}\right)$} \\
\hline Active & $74.8 \pm 12.8$ & $85.7 \pm 10.2$ & $95.1 \pm 10.2$ & $94.1 \pm 10.9$ & $<0.01$ & $T_{2}, T_{3}>T_{1}>T_{0}$ \\
\hline Passive & $80.8 \pm 11.4$ & $91.2 \pm 9.7$ & $104.9 \pm 9.1$ & $103.4 \pm 9.4$ & $<0.01$ & $T_{2}, T_{3}>T_{1}>T_{0}$ \\
\hline \multicolumn{7}{|l|}{ WOMAC score } \\
\hline Stiffness & $75.1 \pm 13.2$ & $27.1 \pm 9.6$ & $19.6 \pm 8.0$ & $18.7 \pm 7.5$ & $<0.01$ & $T_{0}>T_{1}>T_{2}, T_{3}$ \\
\hline Function & $67.3 \pm 12.5$ & $21.5 \pm 8.6$ & $13.9 \pm 7.1$ & $16.1 \pm 6.7$ & $<0.01$ & $T_{0}>T_{1}>T_{3}>T_{2}$ \\
\hline Pain (VAS) & $73.1 \pm 11.8$ & $18.4 \pm 6.7$ & $14.6 \pm 5.8$ & $9.3 \pm 5.5$ & $<0.01$ & $T_{0}>T_{1}>T_{2}>T_{3}$ \\
\hline Beck Depression Scale & $16.4 \pm 9.3$ & $11.3 \pm 3.1$ & $8.0 \pm 2.3$ & $7.8 \pm 2.1$ & $<0.01$ & $T_{0}>T_{1}>T_{2}, T_{3}$ \\
\hline \multicolumn{7}{|l|}{ SF-36 } \\
\hline PCS & $33.7 \pm 8.6$ & $39.2 \pm 6.8$ & $38.8 \pm 6.5$ & $39.7 \pm 7.1$ & $<0.01$ & $T_{3}, T_{1}, T_{2}>T_{0}$ \\
\hline MCS & $44.7 \pm 9.8$ & $51.6 \pm 6.3$ & $50.9 \pm 7.1$ & $50.5 \pm 6.8$ & $<0.01$ & $T_{1}, T_{2}, T_{3}>T_{0}$ \\
\hline
\end{tabular}

Notes: Values are presented as mean \pm standard deviation. Independent samples $t$-test was performed to observe intergroup differences (between intervention and control groups). $* P<0.01$ and $* * P<0.05$. One-way ANOVA with LSD post hoc test was used to assess differences in the intragroup.

Abbreviations: LSD, least significant difference; ROM, range of motion; WOMAC, Western Ontario and McMaster Universities Osteoarthritis Index; VAS, Visual Analog Scale; SF-36, Short Form-36; PCS, physical component summary; MCS, mental component summary; ANOVA, analysis of variance; ANOVAF, one-way analysis of variance; TKA, total knee arthroplasty. 

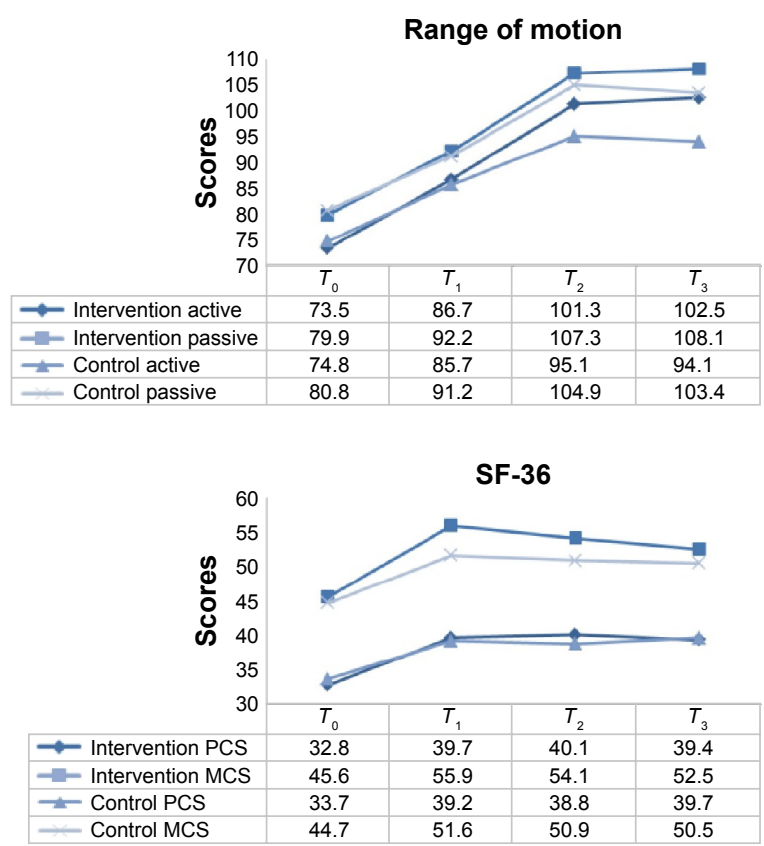

BDS

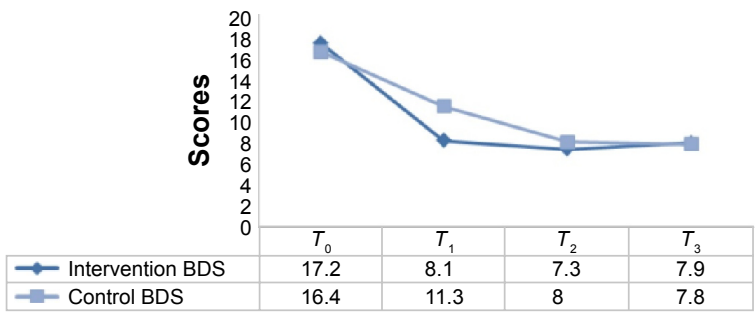

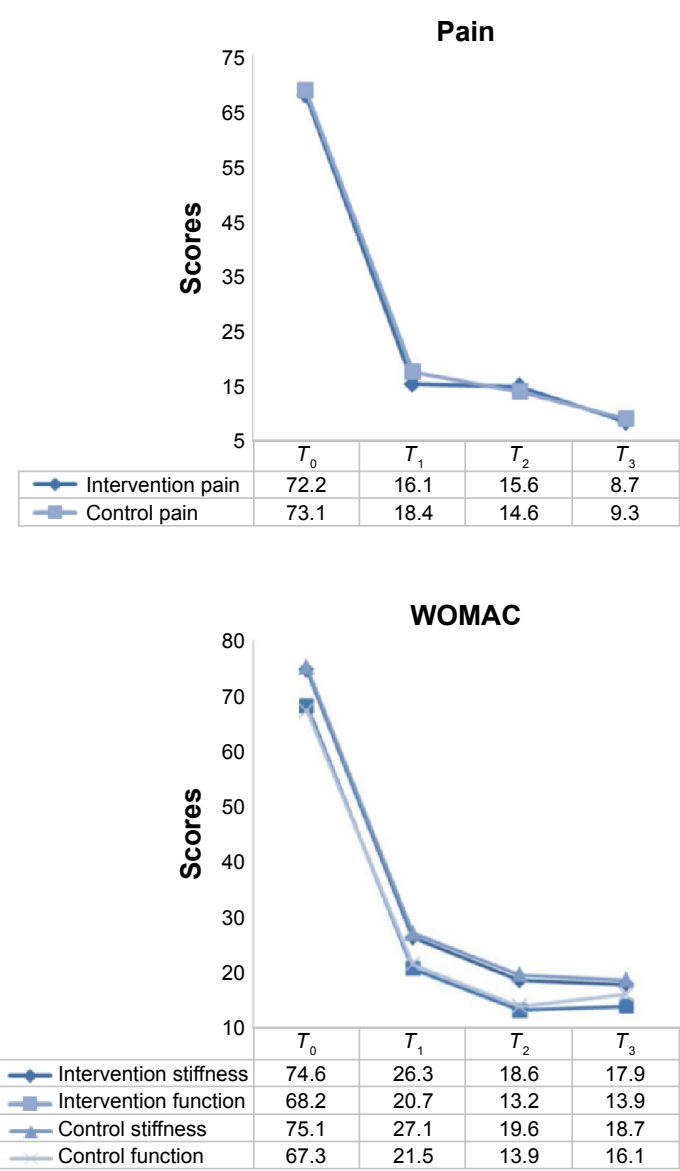

Figure 2 Description of the variable scores 12 months after discharge.

Note: $T_{0}$ represent "before the operation" and $T_{1}, T_{2}, T_{3}$ represent 3, 6, 12 months after the operation, respectively.

Abbreviations: SF-36, Short Form-36; PCS, physical component summary; MCS, mental component summary; WOMAC, Western Ontario and McMaster Universities Osteoarthritis Index; BDS, Beck Depression Scale.

In a previous study about exercise therapy, ${ }^{30}$ improvements in pain, function, stiffness, and ROM after TKA rehabilitation were not related to the intensity and duration of postoperative exercise therapy. The present study agrees with this finding at several special periods. Further, the condition of the

Table 4 Tests of inter-subject effects in a repeated measures analysis of variance

\begin{tabular}{llll}
\hline Variable & df & F & P-value \\
\hline Active ROM & $\mathrm{I}$ & 6.25 & 0.01 \\
Passive ROM & $\mathrm{I}$ & $1.6 \mathrm{I}$ & 0.21 \\
Stiffness (WOMAC) & $\mathrm{I}$ & 0.47 & 0.49 \\
Function (WOMAC) & $\mathrm{I}$ & 0.55 & 0.46 \\
Pain (VAS) & $\mathrm{I}$ & 1.08 & 0.30 \\
Beck depression scale & $\mathrm{I}$ & 0.88 & 0.45 \\
PCS (SF-36) & $\mathrm{I}$ & 0.10 & 0.75 \\
MCS (SF-36) & $\mathrm{I}$ & 4.63 & 0.03 \\
\hline
\end{tabular}

Notes: A P-value $<0.05$ indicates statistical significance. (F) Represent the F-statistic.

Abbreviations: df, degrees of freedom; SF-36, Short Form-36; PCS, physical component summary; MCS, mental component summary; WOMAC, Western Ontario and McMaster Universities Osteoarthritis Index; VAS, Visual Analog Scale; ROM, range of motion. knee changed with time. The results of intersubject effects indicated that structured telephone support was effective at improving the mental health and active ROM of patients for 12 months after TKA. In our clinic, we found that the major reasons for poor patient adherence to home exercises were a lack of an understanding of the benefits of rehabilitation after TKA, forgetting to do the exercise, or complaints of pain with exercise. Structured telephone support could remind the elderly patients to do the exercises, enhance their understanding of the benefits of rehabilitation after TKA, and make them feel more comfortable during rehabilitation. These reasons may explain the improved patient compliance, enhanced mental health, and increased active ROM in our telephone follow-up group.

In summary, this randomized clinical trial explored the concept of structured telephone support as a feasible and acceptable approach to improve patient adherence to home exercises after TKA. However, the present work has a number of potential limitations. First, this study is a singlecenter, randomized, controlled trial. Participants of a certain 
age were recruited from one particular university hospital, and thus, are not representative of the general population. This limited representation may reduce the application of our techniques to the broader public. Second, the duration of the structured telephone support program is only 6 weeks and the follow-up time is only 12 months, which may not be sufficient for a concurrent investigation of the effects of the intervention. Third, individual patient characteristics and other currently unknown factors may influence patient adherence, requiring further investigation. Finally, there was a measurement bias in the participant-reported outcomes because of the varying standards followed by different investigators.

\section{Conclusion}

TKA can significantly relieve pain while improving patient physical function and quality of life. This study demonstrated that structured telephone follow-up can improve patient adherence to home exercises after TKA and patient's mental health and active ROM.

\section{Disclosure}

The authors report no conflicts of interest in this work.

\section{References}

1. Rissanen P, Aro S, Slatis P, Sintonen H, Paavolainen P. Health and quality of life before and after hip or knee arthroplasty. J Arthroplasty. 1995;10(2):169-175.

2. Salmon P, Hall GM, Peerbhoy D, Shenkin A, Parker C. Recovery from hip and knee arthroplasty: patients' perspective on pain, function, quality of life, and well-being up to 6 months postoperatively. Arch Phys Med Rehabil. 2001;82(3):360-366.

3. Ethgen O, Bruyere O, Richy F, Dardennes C, Reginster JY. Healthrelated quality of life in total hip and total knee arthroplasty. A qualitative and systematic review of the literature. J Bone Joint Surg Am. 2004;86-A(5):963-974.

4. Nunez M, Lozano L, Nunez E, et al. Total knee replacement and healthrelated quality of life: factors influencing long-term outcomes. Arthritis Rheum. 2009;61(8):1062-1069.

5. Panel NIHC. NIH consensus statement on total knee replacement December 8-10, 2003. J Bone Joint Surg Am. 2004;86-A(6): $1328-1335$.

6. Steiner ME, Simon SR, Pisciotta JC. Early changes in gait and maximum knee torque following knee arthroplasty. Clin Orthop Relat Res. 1989;(238):174-182.

7. Skinner HB. Pathokinesiology and total joint arthroplasty. Clin Orthop Relat Res. 1993;(288):78-86.

8. Walsh M, Woodhouse LJ, Thomas SG, Finch E. Physical impairments and functional limitations: a comparison of individuals 1 year after total knee arthroplasty with control subjects. Phys Ther. 1998;78(3):248-258.

9. March LM, Cross MJ, Lapsley H, et al. Outcomes after hip or knee replacement surgery for osteoarthritis. A prospective cohort study comparing patients' quality of life before and after surgery with agerelated population norms. Med J Aust. 1999;171(5):235-238.

10. Shields RK, Enloe LJ, Leo KC. Health related quality of life in patients with total hip or knee replacement. Arch Phys Med Rehabil. 1999; 80(5):572-579.
11. Khan F, Ng L, Gonzalez S, Hale T, Turner-Stokes L. Multidisciplinary rehabilitation programmes following joint replacement at the hip and knee in chronic arthropathy. Cochrane Database Syst Rev. 2008;(2): CD004957.

12. Seymour RA, Simpson JM, Charlton JE, Phillips ME. An evaluation of length and end-phrase of visual analogue scales in dental pain. Pain. 1985;21(2):177-185.

13. Norkin CC, White DJ. Measurement of Joint Motion: A Guide to Goniometry. Philadelphia, PA: FA Davis; 2009.

14. Symonds T, Hughes B, Liao S, Ang Q, Bellamy N. Validation of the Chinese Western Ontario and McMaster Universities osteoarthritis index in patients from mainland China with osteoarthritis of the knee. Arthritis Care Res (Hoboken). 2015;67(11):1553-1560.

15. Bellamy N, Buchanan WW, Goldsmith CH, Campbell J, Stitt LW. Validation study of WOMAC: a health status instrument for measuring clinically important patient relevant outcomes to antirheumatic drug therapy in patients with osteoarthritis of the hip or knee. J Rheumatol. 1988;15(12):1833-1840.

16. McConnell S, Kolopack P, Davis AM. The Western Ontario and McMaster Universities osteoarthritis index (WOMAC): a review of its utility and measurement properties. Arthritis Rheum. 2001;45(5): 453-461.

17. Escobar A, Quintana JM, Bilbao A, Arostegui I, Lafuente I, Vidaurreta I. Responsiveness and clinically important differences for the WOMAC and SF-36 after total knee replacement. Osteoarthritis Cartilage. 2007;15(3):273-280.

18. Ware JE Jr, Sherbourne CD. The MOS 36-item short-form health survey (SF-36). I. Conceptual framework and item selection. Med Care. 1992; 30(6):473-483.

19. Li L, Wang HM, Shen Y. Chinese SF-36 health survey: translation, cultural adaptation, validation, and normalisation. J Epidemiol Community Health. 2003;57(4):259-263.

20. Lam CL, Tse EY, Gandek B, Fong DY. The SF-36 summary scales were valid, reliable, and equivalent in a Chinese population. $J$ Clin Epidemiol. 2005;58(8):815-822.

21. Wheatley WB, Krome J, Martin DF. Rehabilitation programmes following arthroscopic meniscectomy in athletes. Sports Med. 1996; 21(6):447-456.

22. Kramer JF, Speechley M, Bourne R, Rorabeck C, Vaz M. Comparison of clinic- and home-based rehabilitation programs after total knee arthroplasty. Clin Orthop Relat Res. 2003;(410):225-234.

23. Rajan RA, Pack Y, Jackson H, Gillies C, Asirvatham R. No need for outpatient physiotherapy following total knee arthroplasty: a randomized trial of 120 patients. Acta Orthop Scand. 2004;75(1):71-73.

24. Patik M, Phillips L, Kladny B, Captain A, Gettig E, Krishnamurti L. Structured telephone-based outreach using nonmedical personnel can improve adherence to comprehensive care in families of children with sickle cell disease. Am J Hematol. 2006;81(6):462-464.

25. Turner D. Can telephone follow-up improve post-discharge outcomes? Br J Nurs. 1996;5(22):1361-1365.

26. Shalaby TM, Orenstein SR. Efficacy of telephone teaching of conservative therapy for infants with symptomatic gastroesophageal reflux referred by pediatricians to pediatric gastroenterologists. J Pediatr. 2003;142(1):57-61.

27. Urien AM, Guillen VF, Beltran DO, et al. Telephonic back-up improves antibiotic compliance in acute tonsillitis/pharyngitis. Int J Antimicrob Agents. 2004;23(2):138-143.

28. Bartlett BW, Firestone AR, Vig KW, Beck FM, Marucha PT. The influence of a structured telephone call on orthodontic pain and anxiety. Am J Orthod Dentofacial Orthop. 2005;128(4):435-441.

29. Lerman C, Daly M, Walsh WP, et al. Communication between patients with breast cancer and health care providers. Determinants and implications. Cancer. 1993;72(9):2612-2620.

30. Zech A, Hendrich S, Pfeifer K. Association between exercise therapy dose and functional improvements in the early postoperative phase after hip and knee arthroplasty: an observational study. PM R. 2015; 7(10):1064-1072. 


\section{Publish your work in this journal}

Patient Preference and Adherence is an international, peer-reviewed, open access journal that focuses on the growing importance of patient preference and adherence throughout the therapeutic continuum. Patient satisfaction, acceptability, quality of life, compliance, persistence and their role in developing new therapeutic modalities and compounds to optimize

clinical outcomes for existing disease states are major areas of interest for the journal. This journal has been accepted for indexing on PubMed Central. The manuscript management system is completely online and includes a very quick and fair peer-review system, which is all easy to use. Visit http://www. dovepress.com/testimonials.php to read real quotes from published authors.

Submit your manuscript here: http://www.dovepress.com/patient-preference-and-adherence-journal 